\title{
Update and Overview of the International League Against Epilepsy Consensus Definition of Drug-resistant Epilepsy
}

\author{
Xiao-Ting Hao, MD¹ and Patrick Kwan, MD, PhD ${ }^{2}$ \\ 1. Doctorate Student, Division of Neurology, Department of Neurology, West China Hospital, Sichuan University School of Medicine; \\ 2. Associate Consultant, Division of Neurology, Department of Medicine and Therapeutics, The Chinese University of Hong Kong, Prince of Wales Hospital
}

\begin{abstract}
Drug-resistant epilepsy remains a major clinical challenge. Diverse criteria have been used to define drug resistance by different researchers, making it difficult or even impossible to compare the results across different studies. To improve patient care and facilitate clinical research, the International League Against Epilepsy (ILAE) recently proposed a consensus definition to define drug-resistant epilepsy. This is the failure of adequate trials of two tolerated, appropriately chosen and used antiepileptic drug schedules (whether as monotherapies or in combination) to achieve sustained seizure freedom. This article outlines the framework of the consensus definition, explains how to apply it in practice, and discusses the future development of its use.
\end{abstract}

\section{Keywords}

Epilepsy, seizures, drug resistant, refractory, pharmacoresistance, antiepileptic drugs (AEDS), definition, International League Against Epilepsy (ILAE)

Disclosure: Xiao-Ting Hao, MD, has no conflicts of interest to declare. Patrick Kwan, MD, PhD, is lead author of the report on the International League Against Epilepsy definition of drug-resistant epilepsy. He has received speaker's or consultancy fees and/or research grants from Eisai, Johnson \& Johnson, Pfizer and UCB Pharma.

Received: October 7, 2010 Accepted: December 2, 2010 Citation: US Neurology, 2010;6(2):122-4 DOI: 10.17925/USN.2010.06.02.122

Correspondence: Patrick Kwan, MD, PhD, Department of Medicine and Therapeutics, Prince of Wales Hospital, Hong Kong. E: patrickkwan@cuhk.edu.hk

Approximately 50 million people have epilepsy worldwide, ${ }^{1}$ up to one-third of whom continue to experience seizures despite drug treatment. ${ }^{2}$ Diverse criteria have been used to define drug resistance by different researchers, making it difficult or even impossible to compare the results across different studies. To improve patients' care and facilitate clinical research, the International League Against Epilepsy (ILAE) recently proposed a consensus definition of drug-resistant epilepsy. Given that most patients are initially managed by general physicians or general neurologists, it is hoped that the definition framework will provide clear and simple guidance in identifying patients with pharmacoresistance for early referral to specialist centers for evaluation. ${ }^{3}$ This article outlines the framework of the consensus definition, explains how to apply it in practice and discusses the future development of its use.

\section{The Burden of Drug-resistant Epilepsy}

Recurrent seizures are associated with a range of deleterious consequences. Seizure-related deaths may account for up to $40 \%$ of all deaths in patients with chronic epilepsy. The rate of sudden unexpected death, which accounts for $7-17 \%$ of deaths among epilepsy patients, is estimated to be up to 27 -fold higher in those with ongoing seizures compared to those who are seizure free. ${ }^{4,5}$ Uncontrolled seizures restrict patients' social activities, reducing their ability to hold a driving license or keep a job. Refractory epilepsy places substantial stress on the patient's family members and caregivers. It is also a great economic burden for society, through expenditures in healthcare and unemployment. In a study of the cost of epilepsy in the US, it was reported that the average montly cost per individual in the patient-based analysis was $\$ 1,490$, whereas the average annual cost per individual in the population-based analysis was $\$ 1,510$ with average yearly costs between $\$ 1,480$ and $\$ 1,740 .{ }^{6}$ In patients whose epilepsy failed to respond to several antiepileptic drugs (AEDs), the chance of significant benefit from a further AED change is estimated to be $<5 \%$ per year. ${ }^{7}$ For these patients, resective surgery is a potential therapeutic option. ${ }^{8}$ Early diagnosis of drug resistance using a universally-accepted definition can facilitate the selection of patients for such non-drug therapies and potentially alleviate the medicosocial and economic burden of refractory epilepsy.

\section{The International League Against Epilepsy Consensus Definition \\ Definition Framework}

The proposal defines drug-resistant epilepsy as failure of adequate trials of two (or more) tolerated, appropriately chosen and used AED schedules (whether as monotherapies or in combination) to achieve sustained seizure freedom. The overall framework of the definition comprises two 'hierarchical' levels. Level 1 provides a general template or scheme to categorize the outcome to each therapeutic intervention (whether pharmacological or non-pharmacological). To categorize the outcome accurately, a minimum dataset of details of the AED history, including the dose and duration the drug was used for, must be available. This is the most important factor in determining whether 
the trial of an intervention is 'informative' in an individual patient. The categories of outcome include 'seizure-free', 'treatment failure', and 'undetermined'. These are further subdivided according to whether the patient experienced adverse effects (see Table 1). Level 1 forms the basis for level 2, which provides a core definition of drug-resistant epilepsy based on two or more 'informative' trials of AEDs resulting in a 'treatment failure' outcome.'

\section{Seizure Freedom and Treatment Failure}

Seizure freedom is defined as freedom from all seizures, including auras, for at least three times the longest pre-treatment interseizure interval or 12 months if the longest pretreatment interseizure interval is less than four months. In the case of persistent seizures, the outcome should be defined as treatment failure. ${ }^{9}$ It should be noted that pretreatment interseizure interval should be defined for each intervention separately. It follows that if the seizures are very infrequent, the patient may need to be followed up for many years to determine outcome. In this case, the longest pre-intervention interseizure interval should be determined from seizures occurring within the preceding 12 months.

\section{Undetermined Outcome and Informative Trial}

Level 1 outcome should be recorded as undetermined if the minimum dataset is unavailable. The minimum dataset contains the details of the intervention history, such as the duration of treatment, the dosage of AEDs and reason for withdrawal (if applicable). In the absence of such information, it cannot be confidently determined whether the epilepsy was truly under control or unresponsive to treatment. In this situation, the outcome to the intervention should be categorized as undetermined. To determine treatment outcome, the AED should have been applied 'adequately'. This may not be the case in some circumstances, for example when an AED is withdrawn due to an allergic rash or is stopped early due to poor tolerability at low dosage. In these situations, the outcome should be considered undetermined. The proposed definition does not specify the dose or duration of each drug that constitutes an 'adequate' trial because this is influenced by a range of intrinsic and extrinsic factors. The definition does, however, require a documented attempt to titrate the dose to a target, clinically-effective dose range. For standardization in research settings, we empirically recommends that the AED should have been used for at least three months at a dose of at least $50 \%$ of the World Health Organization's (WHO's) defined daily dose (DDD). ${ }^{10}$ The DDD is the assumed average maintenance amount for each drug in adults. Obviously, because some patients respond to low doses there is no dosage requirement in defining freedom from seizures.

\section{Values of the Definition}

\section{A Simple and Objective System}

Previous studies have shown that response to the first AED is a powerful prognostic factor. ${ }^{11,12}$ Among patients with epilepsy who failed to respond to two appropriate AEDs, whether as monotherapies or in combination, only $5-10 \%$ would achieve seizure control with a third drug. ${ }^{13-15}$ This rate declines further in subsequent trials. ${ }^{16}$ These observations highlight the prognostic importance of early response to AED treatment. Given that most epilepsy patients are initially managed by non-specialists, the consensus definition is deliberately designed to be a simple and objective system for use by clinicians at all healthcare levels.
Table 1: Scheme for Categorizing Outcome of a Therapeutic Intervention for Epilepsy

\begin{tabular}{lll} 
& & Outcome Category \\
\hline 1 & Seizure-free* & \\
\hline A & Adverse effects: no & 1A \\
\hline B & Adverse effects: yes & $1 \mathrm{~B}$ \\
\hline C & Adverse effects: undetermined & $1 \mathrm{C}$ \\
\hline 2 & Treatment Failure** & \\
\hline A & Adverse effects: no & 2A \\
\hline B & Adverse effects: yes & 2B \\
\hline C & Adverse effects: undetermined & 2C \\
\hline 3 & Undetermined*** & \\
\hline A & Adverse effects: no & $3 \mathrm{~A}$ \\
\hline B & Adverse effects: yes & $3 \mathrm{~B}$ \\
\hline C & Adverse effects: undetermined & $3 \mathrm{C}$ \\
\hline
\end{tabular}

* Seizure freedom is defined as freedom from seizures for a minimum of three times the longest pre-intervention inter-seizure interval or 12 months, whichever is longer. ** Treatment failure is defined as recurrent seizure(s) after the intervention has been adequately applied.

*** Undetermined is defined when the treatment has not been applied adequately for a valid assessment of the outcome or information is lacking to make the assessment. This table is level 1 of the International League Against Epilepsy (ILAE) framework for the definition of drug-resistant epilepsy, reproduced with permission from Elsevier. Source: Kwan P, Brodie $M, 2009 .^{3}$

\section{Information to be Collected During Consultations}

Most patients with drug-resistant epilepsy have a long and complex treatment history. Due to insufficient information, treatments labeled as failures might not have truly failed because they have not been tried adequately due to, for instance, allergic reaction at low dose or early withdrawal for reasons unrelated to treatment. Reported in an abstract, Aparicio and colleagues noted that 27 of 30 patients referred for evaluation of 'drug-resistant epilepsy' did not meet the ILAE definition because of a lack of basic information on AED history provided by the referring neurologists. ${ }^{17}$ Thus, the definition may help clinicians and patients to be alerted to the essential information that needs to be collected during routine consultations when initiating a new AED for categorization of its outcome in the future. Patients should also be educated on the avoidance of triggers of seizure relapse, particularly non-compliance and lifestyle factors, such as sleep deprivation, excessive alcohol intake, an irregular sleep-wake cycle, and drug abuse..$^{13}$

\section{Early Presurgical Evaluation}

Selected patients with drug-resistant epilepsy may benefit from non-pharmacological interventions, such as epilepsy surgery and vagus nerve stimulation. ${ }^{18}$ Given that these interventions are invasive, costly, and not without risk, ${ }^{19}$ confirming the diagnosis of drug resistance is generally considered a prerequisite. There is no consensus definition of drug resistance for the purpose of selecting patients for epilepsy surgery. ${ }^{19-21}$ Diverse criteria used by different groups might have contributed to the disparity in postsurgery outcome reported. ${ }^{22-25}$ By providing the minimum core criteria, the proposed ILAE definition represents a common platform that can be adapted specifically for the purpose of selecting patients for non-drug therapies. This will avoid delay in evaluating patients for these therapeutic options and facilitate meaningful comparison of effectiveness reported in different studies. 


\section{Promotion of a Global Outcome Database}

With a common language in categorizing and defining treatment response, it becomes possible to establish a global database of epilepsy outcomes. Through adopting the same criteria to record information on drug response, research findings from different centers around the world may be compared more directly or even combined for analysis. We believe that such a worldwide database will greatly improve our understanding of the long-term prognosis of epilepsy so that more rational treatment strategies may be formulated.

\section{Future Work}

The proposed definition should not be considered as a fait accompli but a work in progress that should be tested in rigorous prospective studies. Its limitations and areas that need to be refined as new evidence emerges will now be discussed.

\section{Defining an 'Adequate' Drug Trial}

There are multiple internal and external factors that influence the dose required for an 'adequate' trial of an AED, such as:"

- the pharmacological properties of the drug;

- the age of the patient;

- any interaction with concomitant medications; and

- the patient's hepatic and renal functions.

An individualized approach is needed in clinical practice. For the purpose of standardization in the research setting, we suggest that referring to the WHO's DDD may be a reasonable approach. The DDDS are for monotherapy use, however, and might not be applicable for patients taking multiple AEDs that are prone to drug-drug interactions. In addition, the system is intended for use in adults because doses used in children are heavily influenced by body weight. If the DDDs are used, flexibility will be needed. More work is required to determine the most appropriate approach to defining an 'adequate' trial, perhaps by taking into account multiple factors simultaneously.

\section{Classification of Breakthrough Seizures}

It is increasingly recognized that epilepsy may display a fluctuating course in some patients. ${ }^{2}$ Seizures may relapse after a period of prolonged seizure freedom under a variety of circumstances, which may or may not have implications for predicting subsequent outcome. For instance, a patient who experiences a seizure relapse after omitting his usual medications may be expected to regain seizure freedom after improved drug compliance. However, the causal relationship between seizure relapse and external factors, such as sleep deprivation or intercurrent febrile illness is less clear-cut. In the study by Schiller, 25 of 256 seizure-free patients experienced seizure relapse due to 'external reversible triggers' (discontinuation of AED treatment, dose reduction, noncompliance, severe sleep deprivation, high fever). All of these patients were reported to regain seizure remission later. ${ }^{2}$

In the ILAE definition, seizures that occur under external triggers are considered evidence of inadequate seizure control and hence treatment failure, but seizure relapse due to poor treatment compliance or planned dose reduction are not. The validity of this classification needs to be determined in future studies. In addition, there is uncertainty about the most appropriate way to determine the pretreatment interseizure interval if a new AED is initiated after just one breakthrough seizure.

\section{Practical Application and Training}

For its effective and efficient use, the definition of drug-resistant epilepsy should be integrated into the routine medical record system. An electronic system that captures the essential information in defining drug response would greatly facilitate this process and should be promoted as part of an electronic health record system. As multiple parameters are required to categorize treatment outcome and drug responsiveness, software programs that automatically compute the classification may help minimize subjectivity in applying the definition. Training of GPS and neurologists is needed to improve their familiarity with the definition, which will help them refer patients to specialist centers in an appropriate and timely fashion.

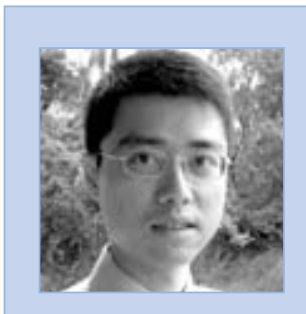

Patrick Kwan, MD, PhD, is an Associate Consultant in the Division of Neurology at the Prince of Wales Hospital in Hong Kong, China. His research interests include the prognosis of epilepsy and the pharmacology of antiepileptic drugs, the mechanisms of drug resistance, co-morbidities, genetics, and pharmacogenomics of epilepsy. He chaired the ad hoc task force of the International League Against Epilepsy to formulate the consensus definition of drug-resistant epilepsy.

Xiao-Ting Hao, MD, is a doctorate student in the Division of Neurology at West China Hospital, Sichuan University School of Medicine in Chengdu, Si Chuan, China. Her research interests include functional magnetic resonance imaging study of epilepsy patients, cognitive effect of antiepileptic drugs, prognosis of epilepsy, and pre-operational validation of drug-resistant epilepsy patients.
1. Neurological Disorders: Public Health Challenges: Global Burden of Neurological Disorders, World Health Organization, 2010. Available at www.who.int/ mental_health/neurology/neurodiso/en/index.html (accessed November 3, 2010).

2. Schiller Y, Arch Neurol, 2009;66:1233-9.

3. Kwan P, Brodie M, Lancet Neurol, 2009;9:27-9.

4. Wannamaker $\mathrm{B}, \mathrm{A}$ perspective on death of persons with epilepsy. Epilepsy and sudden death. In: Lathers $\mathrm{CM}$, Schraeder PL (eds), Epilepsy and Sudden Death, New York, Dekker, 1990:27-37.

5. Tomson T, J Neurol, 2000;247:15-21.

6. Halpern M, Rentz A, Murray M, Neuroepidemiology, 2000;19: 87-99.

7. Mohanraj R, Brodie M, Eur J Neurol, 2006;13:277-82.
8. Sisodiya S, Nat Clin Prac Neurol, 2007;3:320-30

9. Kwan P, Arzimanoglou A, Berg A, et al., Epilepsia, 2010;51:1069-77.

10. World Health Organization Collaborating Centre for Drug Statistics Methodology, About the ATC/DDD System, WHO, 2008.

11. Kwan P, Brodie MJ, N Eng/ J Med, 2000;342:314-9.

12. Dlugos $D$, Sammel M, Strom B, et al., Neurology, 2001;57:2259-64.

13. Perucca E, CNS Drugs, 1998;10:171-9.

14. Kramer G, Epilepsia, 1997;38:S9-S13.

15. Arts W, Geerts A, Brouwer O, et al., Epilepsia, 1999;40: 726-34.

16. French J, Epilepsia, 2007:48(Suppl. 1):3-7.

17. Aparicio Calvo J, Epilepsia, 2010;51:79.
18. Engel Jr J, Wiebe S, French J, et al., Neurology, 2003;60: 538-47.

19. Go C, Snead III O, Neurosurg Focus, 2008;25:E2

20. Unnwongse $\mathrm{K}$, Wehner T, Foldvary-Schaefer N, Curr Neurol Neurosci Rep, 2010;10:299-307.

21. Duncan J, Epilepsy Behav, Aug 13, 2010; [Epub ahead of print].

22. Wass CT, Rajala MM, Hughes JM, et al., Mayo Clin Proc, 1996;71:1105-13.

23. Rougier A, Dartigues J, Commenges D, et al., I Neurol Neurosurg Psychiatry, 1992;55:762-7.

24. Téllez-Zenteno J, Dhar R, Wiebe S, Brain, 2005;128: 1188-98.

25. Jehi L, Sarkis R, Bingaman W, et al., Epilepsia, 2010;51: 994-1003. 